\title{
Efficient isotropic magnetic resonators
}

\author{
Philippe Gay-Balmaz and Olivier J. F. Martin ${ }^{\mathrm{a})}$ \\ Electromagnetic Fields and Microwave Electronics Laboratory, ETHZ, Gloriastrasse 35, \\ 8092 Zurich, Switzerland
}

(Received 26 February 2002; accepted for publication 31 May 2002)

\begin{abstract}
We study experimentally and numerically a novel three-dimensional magnetic resonator structure with high isotropy. It is formed by crossed split-ring resonators and has a response independent of the illumination direction in a specific plane. The utilization of such elements to build a finite left-handed medium is discussed. (C) 2002 American Institute of Physics.
\end{abstract}

[DOI: $10.1063 / 1.1496507$ ]

There is currently growing interest in small magnetic resonant structures. This interest has been spurred by the demonstration that specific subwavelength structures can exhibit effective magnetic permeability which can be tuned to negative values over a finite frequency range, ${ }^{1}$ and by the utilization of these structures to produce a left-handed medium (LHM) in the microwave regime., ${ }^{2,3}$ Thus LHM has negative permittivity $\varepsilon$ and permeability $\mu$, which lead to reversed electromagnetic properties. ${ }^{4}$

Thus far, LHM have been fabricated from periodic arrays of thin wires (to produce a plasmonic medium with $\varepsilon$ $<0),{ }^{5}$ combined with periodic arrays of split-ring resonators (SRRs) to produce $\mu<0 .{ }^{2}$ The SRRs are planar structures that must be arranged in an infinite three-dimensional (3D) lattice to create a LHM. The lattice unit cell consists of three orthogonal SRRs, one along each plane of symmetry. ${ }^{1}$ Such an infinite lattice produces an isotropic magnetic medium, although each SRR is highly anisotropic. By anisotropic, it is meant that the response of the system depends on the direction of illumination. For example, since an isolated SRR mainly reacts to magnetic flux through its structure, it only resonates for specific illumination directions. ${ }^{6}$

The objective of this letter is to propose and study alternative magnetic resonant building blocks with a higher degree of isotropy.

The system we consider is depicted in Fig. 1. It is composed of two intersecting SRRs normal to each other, which form a crossed SRR (CSRR). Each SRR is made of two aluminum (Al) strips deposited on the inner and outer faces of low permittivity foam (Rohacell HF 51, $\varepsilon=1.07$ ). The foam width is $w=4 \mathrm{~mm}$, the inner radius $15 \mathrm{~mm}$ and the outer radius $20 \mathrm{~mm}$ (the separation between inner and outer Al strips is $t=5 \mathrm{~mm}$ ); it is machined from a foam plate to obtain two open rings that are then fitted into each other. The $4 \mathrm{~mm}$ wide Al strips are cut from $50 \mu \mathrm{m}$ thick self-adhesive $\mathrm{Al}$ foil. Note that the gaps at the extremities of the two CSRRs are located at the same poles of the spherical structure (Fig. 1). The width of these gaps is $6 \mathrm{~mm}$.

For our discussion we will designate by $\mathrm{SRR}_{x z}$, respectively, $\mathrm{SRR}_{y z}$ the resonator in the $O x z$, respectively, $O y z$

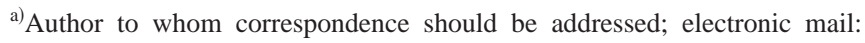
martin@ifh.ee.ethz.ch
}

plane (Fig. 1). Note that the structure in Fig. 1 possesses these two symmetry planes.

Figure 2 shows the scattering parameter $s_{21}$ measured when the structure is placed in a $\mathrm{R} 9$ rectangular waveguide. The waveguide was excited with $\mathrm{TE}_{10}$ mode and $s_{21}$ measured using a HP 8753B network analyzer. We observe a strong narrow resonance at $0.92 \mathrm{GHz}$ (quality factor $Q$ $=250$ ). Rotating the CSRR in the waveguide around its $z$ axis (Fig. 1) does not affect at all the $s_{21}$ measured and the curves associated with the different illumination directions $\varphi$ overlap perfectly.

The structure proposed in Fig. 1 is therefore isotropic in the $O x y$ plane and produces the same response for any illumination direction in this plane.

To further our understanding, the response of the CSRR was simulated with the method of moments. The reader is referred to Ref. 7, where this numerical technique is explained in detail and to Ref. 6, where its utilization for the simulation of individual and coupled planar SRRs is discussed.

Figure 3 shows the three different CSRR geometries that were simulated. Figure 3(a) is the same configuration as that in the experiment, where the gaps at the end of the two crossed SRRs are located on the same pole of the spherical structure [cf. Fig. 3(a) with Fig. 1]. In Fig. 3(b) [Fig. 3(c)], one of the SRR is rotated $90^{\circ},\left(45^{\circ}\right)$. Note that in Fig. 3(b) both gaps are cut by the other ring's strip. However, this structure retains its two $O x z$ and $O y z$ symmetry planes. This

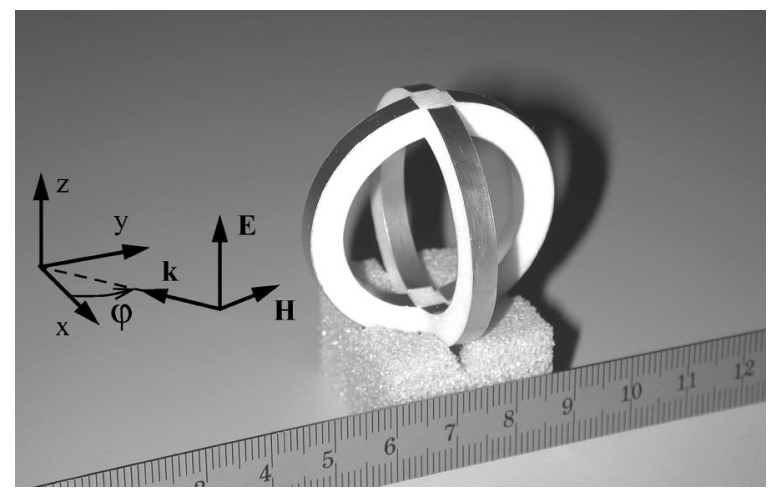

FIG. 1. View of the crossed split-ring resonator. Each SRR is formed by two Al strips deposited on opposite faces of a foam ring. The system is illuminated with a field propagating in the $x y$ plane (incidence angle $\varphi$ ) with the electric and magnetic fields indicated. The scale is in centimeters. 


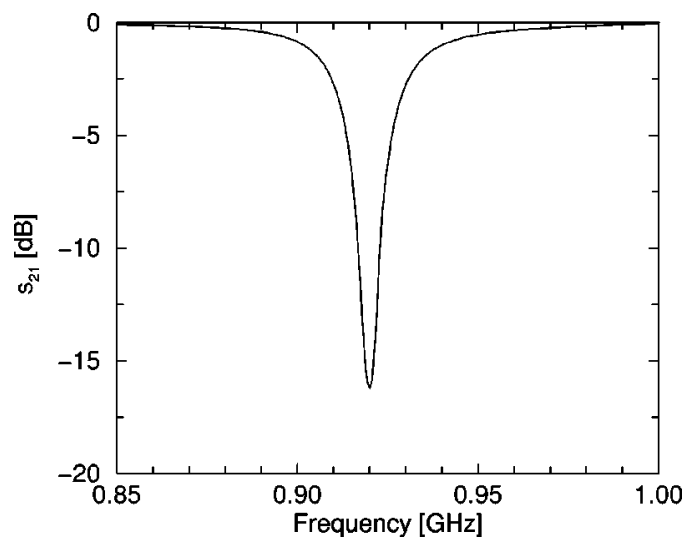

FIG. 2. Scattering parameter $s_{21}$ measured in a R9 waveguide for the structure in Fig. 1.

is not the case in Fig. 3(c), where only one gap is cut by the normal ring's strip.

Figure 4 shows the computed scattering cross section (SCS) associated with the structures in Figs. 3(a) and 3(b). The small discrepancy between the experimental and numerical resonance frequencies is related to the fact that in the experiment the waveguide implicitly imposes periodic boundary conditions on the structure, which shifts the resonance frequency. ${ }^{6}$

The response of the structure in Fig. 3(a) is perfectly isotropic in the $O x y$ plane and when the illumination angle $\varphi$ in that plane changes, the SCS reported in Fig. 4 (solid line) is not at all altered (not shown). This is also the case for the configuration in Fig. 3(b), now with a slightly higher resonance frequency (Fig. 4, dashed line).

On the other hand, the configuration in Fig. 3(c) is no longer isotropic in the $O x y$ plane, or in any other illumination plane. Evidence of this is seen in Fig. 5, where we show the corresponding SCS for different illumination directions $\varphi$ in the $O x y$ plane. Figure 5 shows that one of the SRR that forms the CSRR structure is no longer operating. As discussed in Refs. 1 and 6, a SRR responds to the flux of the magnetic field. For the configuration in Fig. 3(c), the results in Fig. 5 indicate that $\mathrm{SRR}_{y z}$ still responds to the incident field (for example, when $\varphi=90^{\circ}$, only $\mathrm{SRR}_{y z}$ contributes to the resonance since the magnetic field does not penetrate through $\mathrm{SRR}_{x z}$ ). On the other hand, for $\varphi=0$, where $\mathrm{SRR}_{x z}$ should have a strong resonance, we only observe a minute peak in Fig. 5.

These results indicate that the location where the strips of two SRRs intersect determines the resonances properties of the overall structure. We believe that in the configuration in Fig. 3(a), both SRRs intersect at a "neutral point" which
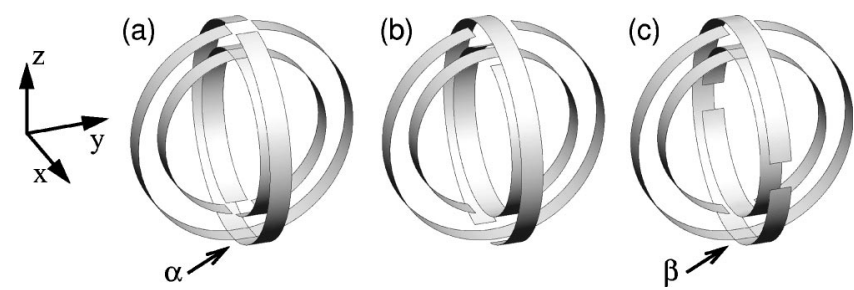

FIG. 3. Three different configurations of CSRRs: (a) same configuration as in the experiment; (b) the $O x z$ SRR is rotated $90^{\circ}$; (c) the $O x z$ SRR is rotated $45^{\circ}$.

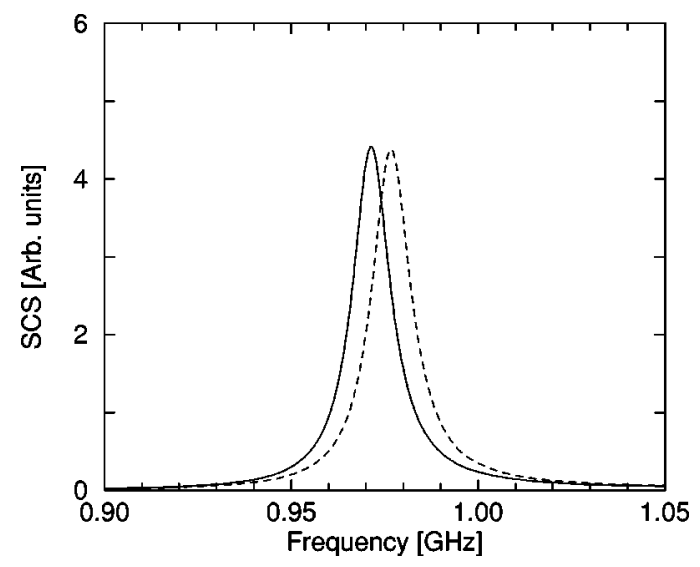

FIG. 4. Computed SCS for the structure in Figs. 3(a) (solid curve) and 3(b) (dashed curve).

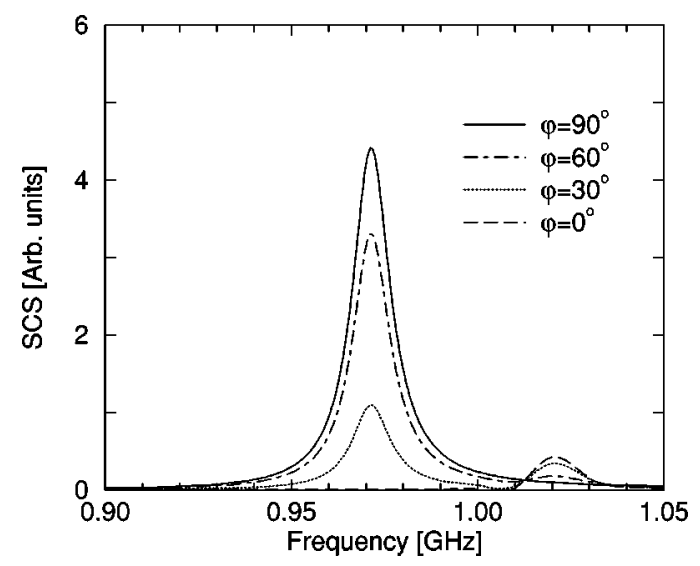

FIG. 5. Computed SCS for the structure in Fig. 3(c) under different illumination angles $\varphi$ in the $O x y$ plane (Fig. 1).
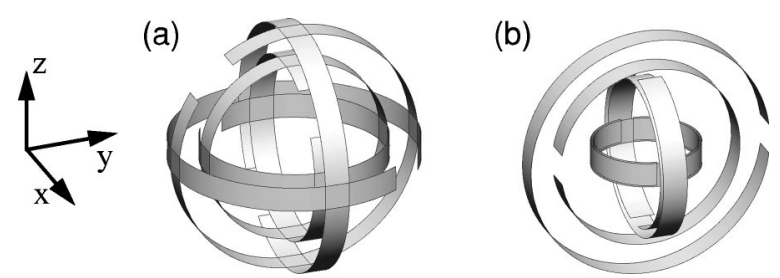

FIG. 6. (a) Structure built from three identical SRRs normal to each other. (b) Structure composed of three SRRs of increasing size.

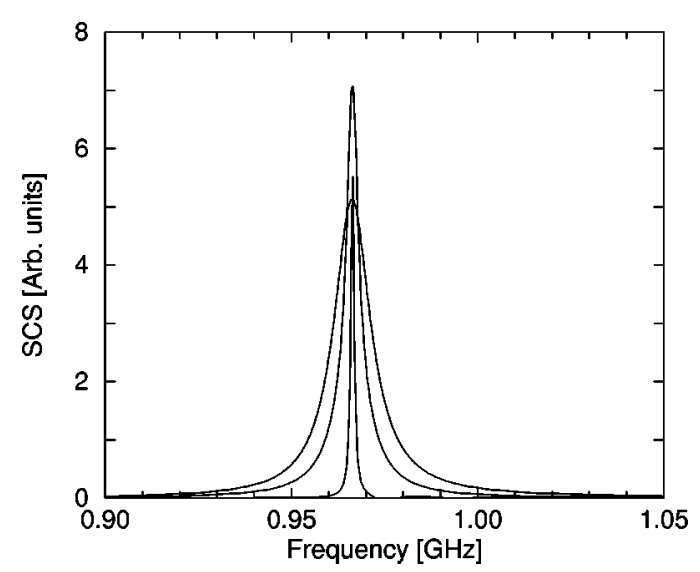

FIG. 7. Computed SCS for the structure in Fig. 6(b) for the three orthogonal illumination directions. 
does not disturb the charge distribution. To illustrate this, let us consider the external strip of the $O x z$ resonator in Fig. 3(a). Plus and minus charges accumulate at the extremities of this strip, on either side of the gap at the top of the structure. By virtue of symmetry, the opposite point at the bottom of the structure (point $\alpha$ in Fig. 3) remains neutral, irrespective of the illumination direction. It is the same for the external strip of the $O y z$ resonator and it does not matter if both strips intersect at this neutral point. A similar situation arises for the inner strips of the CSRR in Fig. 3(a). On the other hand, for the structure in Fig. 3(c), the external strip of $\mathrm{SRR}_{y z}$ intersects that of $\mathrm{SRR}_{x z}$ at a point which is not neutral for $\operatorname{SRR}_{x z}$ (point $\beta$ in Fig. 3). This strongly disturbs $\operatorname{SRR}_{x z}$ which barely resonates anymore (Fig. 5). Since $\operatorname{SRR}_{y z}$ is intersected at its neutral point, its resonance is not affected, as illustrated in Fig. 5 for $\varphi=90^{\circ}$.

The previous results imply that it is not possible to create a fully isotropic CSRR structure from three identical SRRs normal to each other. In such a case, the strip of a resonator will always be intersected at a non-neutral point, as illustrated in Fig. 6(a). This particular geometry does not resonate at all, for any illumination direction.

A possible alternative is the use of three SRRs with varying dimensions, as shown in Fig. 6(b). Unfortunately, the larger a SRR, the lower its resonance frequency. Therefore, to obtain a $3 \mathrm{D}$ structure that resonates at a single frequency for any illumination direction, the three SRRs must be tuned. This can be achieved by a larger distance $t$ between the inner and outer strips, which decreases the capacity, thereby increasing the resonance frequency (our simulations indicate that it is not sufficient to act only on the gap width to tune the resonance frequencies). Regrettably, this in turn broadens the resonance and decreases the $Q$ factor, as illustrated in Fig. 7 (the narrow resonance in Fig. 7 corresponds to the small SRR). However, at the resonance frequency, this structure is perfectly isotropic.
Let us finally come back to the structure in Fig. 3(a), which provides isotropy in the $O_{x y}$ plane. By varying the separation distance $t$ between inner and outer rings, it is possible to strongly increase the computed $Q$ factor: $Q=80(t$ $=5 \mathrm{~mm}), \quad 135(t=3 \mathrm{~mm}), \quad 520(t=1 \mathrm{~mm}), \quad$ and $1200(t$ $=0.5 \mathrm{~mm})$. The resonance frequency is correspondingly redshifted.

To conclude, the CSRR structures proposed in this letter show very strong resonance and perfect isotropy in one illumination plane. By building up an array of such CSRRs with periodic or random orientations, it should be possible to obtain an effective material with isotropic magnetic properties on average.

Finally, the proposed CSRR structure maximizes the $F$ parameter introduced by Pendry et al., ${ }^{1}$ which represents the fractional area of the unit cell occupied by the interior of the split-ring resonator and determines the width of the frequency band over which the effective permeability of the system is negative. Large $F$ values render left-handed effects easier to exploit. ${ }^{8}$

The authors thank C. Maccio and H. Benedickter for their help during the fabrication and measurements. This work was supported by the Swiss National Science Foundation, the Research Board of ETHZ, and AFOSR (Grant No. F496200110440).

${ }^{1}$ J. B. Pendry, A. J. Holden, D. J. Robbins, and W. J. Stewart, IEEE Trans. Microwave Theory Tech. 47, 2075 (1999).

${ }^{2}$ D. R. Smith, W. J. Padilla, D. C. Vier, S. C. Nemat-Nasser, and S. Schultz, Phys. Rev. Lett. 84, 4184 (2000).

${ }^{3}$ R. A. Shelby, D. R. Smith, and S. Schultz, Science 292, 77 (2001).

${ }^{4}$ V. G. Veselago, Sov. Phys. Usp. 10, 509 (1968).

${ }^{5}$ J. B. Pendry, A. J. Holden, W. J. Stewart, and I. Youngs, Phys. Rev. Lett. 76, 4773 (1996).

${ }^{6}$ P. Gay-Balmaz and O. J. F. Martin, J. Appl. Phys. (in press).

${ }^{7}$ P. Gay-Balmaz and J. R. Mosig, Int. J. Microwave Millimeter Wave 3, 330 (1997).

${ }^{8}$ R. Ruppin, Solid State Commun. 116, 411 (2000). 